\title{
Split General Strong Nonlinear Quasi-Variational Inequality Problem
}

\author{
Yali Zhao and Dongxue Han \\ College of Mathematics and Physics, Bohai University, Jinzhou, Liaoning 121013, China \\ Correspondence should be addressed to Yali Zhao; yalizhao2000@163.com
}

Received 27 April 2016; Accepted 11 October 2016

Academic Editor: Hung-Yuan Chung

Copyright ( 2016 Y. Zhao and D. Han. This is an open access article distributed under the Creative Commons Attribution License, which permits unrestricted use, distribution, and reproduction in any medium, provided the original work is properly cited.

\begin{abstract}
We introduce a split general strong nonlinear quasi-variational inequality problem which is a natural extension of a split general quasi-variational inequality problem, split variational inequality problem, and quasi-variational and variational inequality problems in Hilbert spaces. Using the projection method, we propose an iterative algorithm for the split general strongly nonlinear quasivariational inequality problem and discuss the convergence criteria of the iterative algorithm. The results presented here generalized, unify, and improve many previously known results for quasi-variational and variational inequality problems.
\end{abstract}

\section{Introduction}

Variational inequalities are a very powerful tool of the current mathematical technology and have become a rich source of inspiration for scientists and engineers. These have been extended and generalized to study a wide class of problems arising in mechanics, optimization and control problem, operations research and engineering sciences, and so forth. The development of variational inequality theory can be viewed as the simultaneous pursuit of two different lines of research. On one hand, it reveals the fundamental facts on the qualitative behavior of solutions to important classes of problems. On the other hand, it enables us to develop highly efficient and powerful numerical methods to solve, for example, obstacle, unilateral, free, and moving boundary value problems. In the last five decades, considerable interest has been shown in developing various classes of variational inequality problems, both for their own sake and for their applications.

An important generalization of the variational inequality problem is the quasi-variational inequality problem introduced and studied by Bensoussan et al. [1] in connection with impulse control problem. Recently, Kazmi [2] introduced and studied the following split general quasi-variational inequality problem (in short, SpGQVIP). For each $i \in\{1,2\}$, let $H_{i}$ be a Hilbert space with inner product $\langle\cdot, \cdot\rangle$ and induced norm $\|\cdot\|$, let $C_{i}: H_{i} \rightarrow 2^{H_{i}}$ be a nonempty, closed, and convex set-valued mapping, let $f_{i}: H_{i} \rightarrow H_{i}$ and $g_{i}$ : $H_{i} \rightarrow H_{i}$ be nonlinear mappings, and let $A: H_{1} \rightarrow H_{2}$ be a bounded linear operator with its adjoint operator $A^{*}$. Then, the SpGQVIP is to find $x_{1}^{*} \in H_{1}$ such that $g_{1}\left(x_{1}^{*}\right) \in C_{1}\left(x_{1}^{*}\right)$ and

$$
\left\langle f_{1}\left(x_{1}^{*}\right), x_{1}-g_{1}\left(x_{1}^{*}\right)\right\rangle \geq 0 \quad \forall x_{1} \in C_{1}\left(x_{1}^{*}\right)
$$

and such that, $x_{2}^{*}=A x_{1}^{*} \in H_{2}, g_{2}\left(x_{2}^{*}\right) \in C_{2}\left(x_{2}^{*}\right)$ solves

$$
\left\langle f_{2}\left(x_{2}^{*}\right), x_{2}-g_{2}\left(x_{2}^{*}\right)\right\rangle \geq 0 \quad \forall x_{2} \in C_{2}\left(x_{2}^{*}\right) .
$$

SpGQVIP (1a)-(1b) amounts to saying the following: find a solution of general quasi-variational inequality GQVI (1a) whose image under a given bounded linear operator is a solution of GQVIP (1b). If $g_{i}=I_{i}$, where $I_{i}$ is an identity mapping on $H_{i}, C_{i}\left(x_{i}\right)=C_{i}$ for all $x_{i} \in H_{i}$, and then SpGQVIP (1a)-(1b) is reduced to the following SpVIP. Find $x_{1}^{*} \in C_{1}$ such that

$$
\left\langle f_{1}\left(x_{1}^{*}\right), x_{1}-x_{1}^{*}\right\rangle \geq 0 \quad \forall x_{1} \in C_{1}
$$

and such that $x_{2}^{*}=A x_{1}^{*} \in C_{2}$ solves

$$
\left\langle f_{2}\left(x_{2}^{*}\right), x_{2}-x_{2}^{*}\right\rangle \geq 0 \quad \forall x_{2} \in C_{2} .
$$


SpVIP (2a)-(2b) has been introduced and studied by Censor et al. [3]. It is worth mentioning that the SpVIP (2a)-(2b) is quite general and permits split minimization between two spaces so that the imagine of a minimizer of a given function, under a bounded linear operator, is a minimizer of another function and it includes as a special case the split zero problem and the split feasibility problem which have already been studied and used in practice as a model in the intensity-modulated radiation therapy planning; see [4-6] and the references therein.

In this paper, we introduced the following split general strongly nonlinear quasi-variational inequality problem: for each $i \in\{1,2\}$, let $C_{i}: H_{i} \rightarrow 2^{H_{i}}$ be a nonempty, closed, and convex set-valued mapping, let $f_{i}: H_{i} \rightarrow H_{i}, h_{i}: H_{i} \rightarrow$ $H_{i}$, and $g_{i}: H_{i} \rightarrow H_{i}$ be three nonlinear mappings, and let $A: H_{1} \rightarrow H_{2}$ be a bounded linear operator with its adjoint operator $A^{*}$. Then, we consider the problem: find $x_{1}^{*} \in H_{1}$ such that $g_{1}\left(x_{1}^{*}\right) \in C_{1}\left(x_{1}^{*}\right)$ and

$$
\begin{aligned}
&\left\langle f_{1}\left(x_{1}^{*}\right)-h_{1}\left(x_{1}^{*}\right), x_{1}-g_{1}\left(x_{1}^{*}\right)\right\rangle \geq 0 \\
& \forall x_{1} \in C_{1}\left(x_{1}^{*}\right)
\end{aligned}
$$

and such that $x_{2}^{*}=A x_{1}^{*} \in H_{2}, g_{2}\left(x_{2}^{*}\right) \in C_{2}\left(x_{2}^{*}\right)$ solves

$$
\begin{aligned}
\left\langle f_{2}\left(x_{2}^{*}\right)-h_{2}\left(x_{2}^{*}\right), x_{2}-g_{2}\left(x_{2}^{*}\right)\right\rangle \geq 0 & \\
& \forall x_{2} \in C_{2}\left(x_{2}^{*}\right) .
\end{aligned}
$$

We call problem (3a)-(3b) the split general strongly nonlinear quasi-variational inequality problem (in short, SpGSNQVIP).

Remark 1. If $h_{i}=0$, then SpGSNQVIP (3a)-(3b) is reduced to SpGQVIP (1a)-(1b). So SpGSNQVIP (3a)-(3b) is the generalization of SpGQVIP (1a)-(1b).

Remark 2. Note that general strongly nonlinear variational inequality problem $\left\langle f_{1}\left(x_{1}^{*}\right)-h_{1}\left(x_{1}^{*}\right), x_{1}-x_{1}^{*}\right\rangle \geq 0, \forall x_{1} \in C_{1}$, is an important class of variational inequalities, which is the optimal condition of the following minimization problem:

$$
\min _{x \in C}\left(\frac{1}{2}\left\langle f_{1}(x), x\right\rangle-T_{1}(x)\right)
$$

where $T_{1}^{\prime}=h_{1}(x)$. We denote the solution set of SpGSNQVIP (3a)-(3b) and the solution set of SpGQVIP (1a)-(1b) by $\Gamma_{1}$ and $\Gamma_{2}$, respectively.

Example 3. Letting $H_{1}=H_{2}=R, C_{1}\left(x_{1}\right)=[2,5]\left(\forall x_{1} \in\right.$ $\left.H_{1}\right)$, and $C_{2}\left(x_{2}\right)=[1,4]\left(\forall x_{2} \in H_{2}\right)$,

$$
\begin{aligned}
& f_{1}\left(x_{1}\right)=2 x_{1}, \\
& h_{1}\left(x_{1}\right)=3 x_{1}, \\
& f_{2}\left(x_{2}\right)=-x_{2}, \\
& h_{2}\left(x_{2}\right)=-2 x_{2},
\end{aligned}
$$$$
\forall x_{2} \in H_{2}
$$

$$
\begin{aligned}
& g_{1}(x)=g_{2}(x)=x, \quad \forall x \in H_{1}=H_{2} ; \\
& A(x)=\frac{1}{5} x, \quad \forall x \in H_{1} .
\end{aligned}
$$

It is easy to see that $x_{1}^{*}=5$ satisfies ( $\left.3 a\right)$ and $A(5)=1$ satisfies (3b). So $5 \in \Gamma_{1}$ and thus $\Gamma_{1} \neq \varnothing$.

Example 4. Let $H_{1}=R$ with the norm $|\cdot|$ and $H_{2}=R^{2}$ with the norm

$$
\|y\|=\left(y_{1}^{2}+y_{2}^{2}\right)^{1 / 2}, \quad \forall y=\left(y_{1}, y_{2}\right) \in R^{2} .
$$

Set

$$
\begin{aligned}
& C_{1}(x)=[2,+\infty) \quad\left(\forall x \in H_{1}\right), \\
& C_{2}(y)=\left\{y=\left(y_{1}, y_{2}\right) \in R^{2} \mid y_{2} \geq y_{1}+1\right\}
\end{aligned}
$$$$
\left(\forall y \in H_{2}\right)
$$

$$
\begin{aligned}
& f_{1}(x)=3 x, \\
& h_{1}(x)=2 x, \\
& f_{2}(y)=\left(\frac{y_{1}}{2}, \frac{y_{2}}{2}\right), \\
& h_{2}(y)=\left(\frac{y_{2}}{2}, \frac{y_{1}}{2}\right), \\
& g_{1}(x)=x \quad\left(\forall x \in H_{1}\right), \\
& g_{2}(y)=y \quad\left(\forall y \in H_{2}\right) .
\end{aligned}
$$

It is easy to see that the solution sets of ( $3 \mathrm{a})$ and ( $3 \mathrm{~b})$ are $S_{1}=$ $\{2\}$ and $S_{2}=\left\{y^{*}=\left(y_{1}^{*}, y_{2}^{*}\right) \mid y_{2}^{*}-y_{1}^{*}=1\right\}$, respectively. Letting $A x=(x,(3 / 2) x)\left(\forall x \in H_{1}\right)$, then $A: H_{1} \rightarrow H_{2}$ is a bounded linear operator. It is obvious that if $x^{*}=2 \in S_{1}$, then $A\left(x^{*}\right)=(2,3) \in S_{2}$, and so $2 \in \Gamma_{1} \neq \varnothing$.

In this paper, using the projection method, we propose an iterative algorithm for SpGSNQVIP (3a)-(3b) and discuss the convergence of the iterative algorithm. The results presented here generalized, unify, and improve the many previously known results for quasi-variational and variational inequality problems.

\section{Iterative Algorithms and Convergence Results}

For each $i \in\{1,2\}$, a mapping $P_{C_{i}}$ is said to be the metric projection of $H_{i}$ on $C_{i}$ if, for every point $x_{i} \in H_{i}$, there exists a unique nearest point in $C_{i}$ denoted by $P_{C_{i}}\left(x_{i}\right)$ such that

$$
\left\|x_{i}-P_{C_{i}}\left(x_{i}\right)\right\| \leq\left\|x_{i}-y_{i}\right\| \quad \forall y_{i} \in C_{i} .
$$

It is well known that $P_{C_{i}}$ is nonexpansive and satisfies

$$
\left\langle x_{i}-y_{i}, P_{C_{i}}\left(x_{i}\right)-P_{C_{i}}\left(y_{i}\right)\right\rangle \geq\left\|P_{C_{i}}\left(x_{i}\right)-P_{C_{i}}\left(y_{i}\right)\right\|^{2}
$$


Moreover, $P_{C_{i}}\left(x_{i}\right)$ is characterized by

$$
\left\langle x_{i}-P_{C_{i}}\left(x_{i}\right), y_{i}-P_{C_{i}}\left(x_{i}\right)\right\rangle \leq 0 \quad \forall y_{i} \in C_{i} .
$$

Further, it is easy to see the following fact: $x_{1}^{*}$ satisfied QVIP $\Leftrightarrow$ we find $x_{1}^{*} \in C_{1}\left(x_{1}^{*}\right)$ such that

$$
\begin{aligned}
\left\langle f_{1}\left(x_{1}^{*}\right), x_{1}-x_{1}^{*}\right\rangle & \geq 0 \quad\left(\forall x_{1} \in C_{1}\left(x_{1}^{*}\right)\right) \Longleftrightarrow \\
x_{1}^{*} & =P_{C_{1}\left(x_{1}^{*}\right)}\left(x_{1}^{*}-\rho_{1} f_{1}\left(x_{1}^{*}\right)\right),
\end{aligned}
$$

$$
\rho_{1}>0 \text {. }
$$

Hence, SpGSNQVIP (3a)-(3b) can be reformulated as follows: find $x_{1}^{*} \in H_{1}$ with $x_{2}^{*}=A x_{1}^{*}$ such that $g_{i}\left(x_{i}^{*}\right) \in C_{i}\left(x_{i}^{*}\right)$ and

$$
g_{i}\left(x_{i}^{*}\right)=P_{C_{i}\left(x_{i}^{*}\right)}\left[g_{i}\left(x_{i}^{*}\right)-\rho_{i}\left(f_{i}\left(x_{i}^{*}\right)-h_{i}\left(x_{i}^{*}\right)\right)\right]
$$

for $\rho_{i}>0$.

Based on the above-mentioned arguments, we propose the following iterative algorithm for approximating a solution to SpGSNQVIP (3a)-(3b).

Let $\left\{\alpha^{n}\right\} \subset(0,1)$ be a sequence such that $\sum_{n=1}^{\infty} \alpha_{n}=\infty$, and let $\rho_{1}, \rho_{2}$, and $\gamma$ be the parameters with positive values.

Algorithm 5. Given $x_{1}^{0} \in H_{1}$, compute the iterative sequence $\left\{x_{1}^{n}\right\}$ by the iterative schemes:

$$
\begin{aligned}
& g_{1}\left(y^{n}\right)=P_{C_{1}\left(x_{1}^{n}\right)}\left[g_{1}\left(x_{1}^{n}\right)-\rho_{1}\left(f_{1}\left(x_{1}^{n}\right)-h_{1}\left(x_{1}^{n}\right)\right)\right], \\
& g_{2}\left(z^{n}\right) \\
& \quad=P_{C_{2}\left(A y^{n}\right)}\left[g_{2}\left(A y^{n}\right)-\rho_{2}\left(f_{2}\left(A y^{n}\right)-h_{2}\left(A y^{n}\right)\right)\right], \\
& x_{1}^{n+1}=\left(1-\alpha_{n}\right) x_{1}^{n}+\alpha_{n}\left[y^{n}+\gamma A^{*}\left(z^{n}-A y^{n}\right)\right]
\end{aligned}
$$

for all $n=0,1,2, \ldots, \rho_{1}, \rho_{2}, \gamma>0$.

If $h_{i}=0$, then Algorithm 5 is reduced to the following iterative algorithm for SpGQVIP (1a)-(1b).

Algorithm 6. Given $x_{1}^{0} \in H_{1}$, compute the iterative sequence $\left\{x_{1}^{n}\right\}$ by the iterative schemes:

$$
\begin{aligned}
g_{1}\left(y^{n}\right) & =P_{C_{1}\left(x_{1}^{n}\right)}\left[g_{1}\left(x_{1}^{n}\right)-\rho_{1} f_{1}\left(x_{1}^{n}\right)\right], \\
g_{2}\left(z^{n}\right) & =P_{C_{2}\left(A y^{n}\right)}\left[g_{2}\left(A y^{n}\right)-\rho_{2} f_{2}\left(A y^{n}\right)\right], \\
x_{1}^{n+1} & =\left(1-\alpha_{n}\right) x_{1}^{n}+\alpha_{n}\left[y^{n}+\gamma A^{*}\left(z^{n}-A y^{n}\right)\right]
\end{aligned}
$$

for all $n=0,1,2, \ldots, \rho_{1}, \rho_{2}, \gamma>0$.

If $g_{i}=I_{i}, C_{i}\left(x_{i}\right)=C_{i}\left(\forall x_{i} \in H_{i}\right)$, where $C_{i}$ is a nonempty closed convex subset of $H_{i}$, and then Algorithm 5 is reduced to the following iterative algorithm for SpVIP (2a)-(2b).

Algorithm 7. Given $x_{1}^{0} \in H_{1}$, compute the iterative sequence $\left\{x_{1}^{n}\right\}$ by the iterative schemes:

$$
\begin{aligned}
y^{n} & =P_{C_{1}}\left[x_{1}^{n}-\rho_{1} f_{1}\left(x_{1}^{n}\right)\right], \\
z^{n} & =P_{C_{2}}\left[A y^{n}-\rho_{2} f_{2}\left(A y^{n}\right)\right], \\
x_{1}^{n+1} & =\left(1-\alpha_{n}\right) x_{1}^{n}+\alpha_{n}\left[y^{n}+\gamma A^{*}\left(z^{n}-A y^{n}\right)\right]
\end{aligned}
$$

for all $n=0,1,2, \ldots, \rho_{1}, \rho_{2}, \gamma>0$.
Remark 8. Algorithms 6 and 7 are proposed by Kazmi in [2] and [7], respectively. Note that Algorithm 5 concludes them as special cases.

In order to obtain our main results, we need the following assumption, definition, and lemmas.

Assumption 9. For all $x_{i}, y_{i}, z_{i} \in H_{i}$, the operator $P_{C_{i}\left(x_{i}\right)}$ satisfies the following condition:

$$
\left\|P_{C_{i}\left(x_{i}\right)}\left(z_{i}\right)-P_{C_{i}\left(y_{i}\right)}\left(z_{i}\right)\right\| \leq v_{i}\left\|x_{i}-y_{i}\right\|
$$

for some constant $v_{i}>0$.

Definition 10. A nonlinear mapping $f_{1}: H_{1} \rightarrow H_{1}$ is said to be

(i) $\alpha_{1}$-strongly monotone with respect to $g_{1}: H_{1} \rightarrow H_{1}$ if there exists a constant $\alpha_{1}>0$ such that

$$
\begin{array}{r}
\left\langle f_{1}(x)-f_{1}(y), g_{1}(x)-g_{1}(y)\right\rangle \geq \alpha_{1}\|x-y\|^{2}, \\
\forall x, y \in H_{1},
\end{array}
$$

(ii) $\beta_{1}$-Lipschitz continuous if there exists a constant $\beta_{1}>$ 0 such that

$$
\left\|f_{1}(x)-f_{1}(y)\right\| \leq \beta_{1}\|x-y\|, \quad \forall x, y \in H
$$

Remark 11. If $g_{1}=I_{1}$, where $I_{1}$ is an identity mapping on $H_{1}$, then Definition 10(i) is reduced to the definition of $\alpha_{1}$-strong monotonicity of $f$.

Lemma 12. Let $H$ be a real Hilbert space. Then, the following inequalities hold:
(1) $\|x+y\|^{2} \leq\|x\|^{2}+2\langle y, x+y\rangle, \forall x, y \in H$,
(2) $\langle x, y\rangle=(1 / 2)\left(\|x\|^{2}+\|y\|^{2}-\|x-y\|^{2}\right), \forall x, y \in H$.

Lemma 13 (see [8]). Assume that $\left\{a_{n}\right\}$ is a sequence of nonnegative numbers such that $a_{n+1} \leq\left(1-\gamma_{n}\right) a_{n}+\delta_{n}$, where $\left\{\gamma_{n}\right\}$ is a sequence in $(0,1)$ and $\left\{\delta_{n}\right\}$ is a sequence such that

(i) $\sum_{n=1}^{\infty} \gamma_{n}=\infty$;

(ii) $\lim \sup _{n \rightarrow \infty}\left(\delta_{n} / \gamma_{n}\right) \leq 0$

or $\sum_{n=1}^{\infty}\left|\delta_{n}\right|<\infty$; then $\lim _{n \rightarrow \infty} a_{n}=0$.

Now we study the convergence of Algorithm 5 for SpGSNQVIP (3a)-(3b).

Theorem 14. For each $i \in\{1,2\}$, let $C_{i}: H_{i} \rightarrow 2^{H_{i}}$ be a nonempty, closed, and convex set-valued mapping, and let $g_{i}: H_{i} \rightarrow H_{i}$ be $\delta_{i}$-Lipschitz continuous such that $\left(g_{i}-I_{i}\right)$ is $\sigma_{i}$-strongly monotone, where $I_{i}$ is the identity mapping on $H_{i}$. Let $f_{i}: H_{i} \rightarrow H_{i}$ be $\alpha_{i}$-strongly monotone with respect to $g_{i}$ and $\beta_{i}$-Lipschitz continuous. Let $h_{i}: H_{i} \rightarrow H_{i}$ be $\xi_{i}$-Lipschitz continuous and let $A: H_{1} \rightarrow H_{2}$ be a bounded linear operator and $A^{*}$ be its adjoint mapping. Suppose $x_{1}^{*} \in H_{1}$ is a solution to SpGSNQVIP (3a)-(3b) and Assumption 9 holds. Then, the 
sequence $\left\{x_{1}^{n}\right\}$ generated by Algorithm 5 convergences strongly to $x_{1}^{*}$ provided that the constants $\rho_{i}$ and $\gamma$ satisfy the following conditions:

$$
\begin{aligned}
& \left|\rho_{1}-\frac{\alpha_{1}-k_{1} \xi_{1}}{\beta_{1}^{2}-\xi_{1}^{2}}\right| \\
& \leq \frac{\sqrt{\left(\alpha_{1}-k_{1} \xi_{1}\right)^{2}-\left(\delta_{1}{ }^{2}-k_{1}{ }^{2}\right)\left(\beta_{1}{ }^{2}-\xi_{1}{ }^{2}\right)}}{\beta_{1}{ }^{2}-\xi_{1}{ }^{2}}, \\
& k_{1}=\frac{\sqrt{1+2 \sigma_{1}}}{1+2 \theta_{2}}-v_{1} \text {, } \\
& \left|\alpha_{1}-k_{1} \xi_{1}\right|>\sqrt{\left(\delta_{1}^{2}-k_{1}^{2}\right)\left({\beta_{1}}^{2}-\xi_{1}^{2}\right)}, \\
& \delta_{1}>\left|k_{1}\right|, \beta_{1}>\xi_{1}, \\
& 0<\theta_{2} \\
& =\frac{1}{\sqrt{1+2 \sigma_{2}}}\left\{v_{2}+\sqrt{\delta_{2}-2 \rho_{2} \alpha_{2}+\rho_{2}{ }^{2} \beta_{2}^{2}}+\rho_{2} \xi_{2}\right\}, \\
& \rho_{2}>0, \gamma \in\left(0, \frac{2}{\|A\|^{2}}\right) \text {. }
\end{aligned}
$$

Proof. Since $x_{1}^{*} \in H_{1}$ is a solution of SpGSNQVIP (3a)-(3b), $x_{1}^{*} \in H_{1}$ such that $g_{i}\left(x_{i}^{*}\right) \in C_{i}\left(x_{i}^{*}\right)$ and

$$
\begin{aligned}
& g_{1}\left(x_{1}^{*}\right)=P_{C_{1}\left(x_{1}^{*}\right)}\left[g_{1}\left(x_{1}^{*}\right)-\rho_{1}\left(f_{1}\left(x_{1}^{*}\right)-h_{1}\left(x_{1}^{*}\right)\right)\right], \\
& g_{2}\left(A x_{1}^{*}\right) \\
& \quad=P_{C_{2}\left(A x_{1}^{*}\right)}\left[g_{2}\left(A x_{1}^{*}\right)-\rho_{2}\left(f_{2}\left(A x_{1}^{*}\right)-h_{2}\left(A x_{1}^{*}\right)\right)\right]
\end{aligned}
$$

for $\rho_{i}>0$. It follows from Algorithm 5(13a), Assumption 9, and (20) that

$$
\begin{aligned}
& \left\|g_{1}\left(y^{n}\right)-g_{1}\left(x_{1}^{*}\right)\right\| \\
& \quad=\| P_{C_{1}\left(x_{1}^{n}\right)}\left[g_{1}\left(x_{1}^{n}\right)-\rho_{1}\left(f_{1}\left(x_{1}^{n}\right)-h_{1}\left(x_{1}^{n}\right)\right)\right] \\
& \quad-P_{C_{1}\left(x_{1}^{*}\right)}\left[g_{1}\left(x_{1}^{*}\right)-\rho_{1}\left(f_{1}\left(x_{1}^{*}\right)-h_{1}\left(x_{1}^{*}\right)\right)\right] \| \\
& \quad \leq \| P_{C_{1}\left(x_{1}^{n}\right)}\left[g_{1}\left(x_{1}^{n}\right)-\rho_{1}\left(f_{1}\left(x_{1}^{n}\right)-h_{1}\left(x_{1}^{n}\right)\right)\right] \\
& \quad-P_{C_{1}\left(x_{1}^{n}\right)}\left[g_{1}\left(x_{1}^{*}\right)-\rho_{1}\left(f_{1}\left(x_{1}^{*}\right)-h_{1}\left(x_{1}^{*}\right)\right)\right] \| \\
& \quad+\| P_{C_{1}\left(x_{1}^{n}\right)}\left[g_{1}\left(x_{1}^{*}\right)-\rho_{1}\left(f_{1}\left(x_{1}^{*}\right)-h_{1}\left(x_{1}^{*}\right)\right)\right] \\
& \quad-P_{C_{1}\left(x_{1}^{*}\right)}\left[g_{1}\left(x_{1}^{*}\right)-\rho_{1}\left(f_{1}\left(x_{1}^{*}\right)-h_{1}\left(x_{1}^{*}\right)\right)\right] \| \\
& \quad \leq\left\|g_{1}\left(x_{1}^{n}\right)-g_{1}\left(x_{1}^{*}\right)-\rho_{1}\left(f_{1}\left(x_{1}^{n}\right)-f_{1}\left(x_{1}^{*}\right)\right)\right\| \\
& \quad+\rho_{1}\left\|h_{1}\left(x_{1}^{n}\right)-h_{1}\left(x_{1}^{*}\right)\right\|+v_{1}\left\|x_{1}^{n}-x_{1}^{*}\right\| .
\end{aligned}
$$

Noting that $f_{1}$ is $\alpha_{1}$-strongly monotone with respect to $g_{1}$ and $\beta_{1}$-Lipschitz continuous and $g_{1}$ is $\delta_{1}$-Lipschitz continuous, we have

$$
\begin{aligned}
&\left\|g_{1}\left(x_{1}^{n}\right)-g_{1}\left(x_{1}^{*}\right)-\rho_{1}\left(f_{1}\left(x_{1}^{n}\right)-f_{1}\left(x_{1}^{*}\right)\right)\right\|^{2} \\
&=\left\|g_{1}\left(x_{1}^{n}\right)-g_{1}\left(x_{1}^{*}\right)\right\|^{2} \\
& \quad-2 \rho_{1}\left\langle f_{1}\left(x_{1}^{n}\right)-f_{1}\left(x_{1}^{*}\right), g_{1}\left(x_{1}^{n}\right)-g_{1}\left(x_{1}^{*}\right)\right\rangle \\
&+\rho_{1}^{2}\left\|f_{1}\left(x_{1}^{n}\right)-f_{1}\left(x_{1}^{*}\right)\right\|^{2} \\
& \leq\left(\delta_{1}^{2}-2 \rho_{1} \alpha_{1}+\rho_{1}^{2}{\beta_{1}}^{2}\right)\left\|x_{1}^{n}-x_{1}^{*}\right\|^{2} .
\end{aligned}
$$

Combining (22) and (23), we get

$$
\begin{aligned}
& \left\|g_{1}\left(y^{n}\right)-g_{1}\left(x_{1}^{*}\right)\right\| \\
& \quad \leq\left\{\sqrt{\delta_{1}{ }^{2}-2 \rho_{1} \alpha_{1}+\rho_{1}{ }^{2} \beta_{1}{ }^{2}}+\rho_{1} \xi_{1}+v_{1}\right\}\left\|x_{1}^{n}-x_{1}^{*}\right\| .
\end{aligned}
$$

Since $\left(g_{1}-I_{1}\right)$ is $\sigma_{1}$-strongly monotone, by virtue of Lemma 12(1), we have

$$
\begin{aligned}
\left\|y^{n}-x_{1}^{*}\right\|^{2} & \\
\leq & \left\|g_{1}\left(y^{n}\right)-g_{1}\left(x_{1}^{*}\right)\right\|^{2} \\
& \quad-2\left\langle\left(g_{1}-I_{1}\right) y^{n}-\left(g_{1}-I_{1}\right) x_{1}^{*}, y^{n}-x_{1}^{*}\right\rangle \\
\leq & \left\|g_{1}\left(y^{n}\right)-g_{1}\left(x_{1}^{*}\right)\right\|^{2}-2 \sigma_{1}\left\|y^{n}-x_{1}^{*}\right\|^{2},
\end{aligned}
$$

which implies that

$$
\left\|y^{n}-x_{1}^{*}\right\|^{2} \leq \frac{1}{\sqrt{1+2 \sigma_{1}}}\left\|g_{1}\left(y^{n}\right)-g_{1}\left(x_{1}^{*}\right)\right\| .
$$

It follows from (24) and (26) that we have

$$
\left\|y^{n}-x_{1}^{*}\right\| \leq \theta_{1}\left\|x_{1}^{n}-x_{1}^{*}\right\|,
$$

where $\theta_{1}=\left(1 / \sqrt{1+2 \sigma_{1}}\right)\left\{\sqrt{\delta_{1}^{2}-2 \rho_{1} \alpha_{1}+\rho_{1}^{2} \beta_{1}^{2}}+\rho_{1} \xi_{1}+v_{1}\right\}$. Similarly, we obtain

$$
\begin{aligned}
& \left\|g_{2}\left(z^{n}\right)-g_{2}\left(A x_{1}^{*}\right)\right\| \\
& \quad \leq\left\{\sqrt{\delta_{2}^{2}-2 \rho_{2} \alpha_{2}+\rho_{2}^{2} \beta_{2}^{2}}+\rho_{2} \xi_{2}+v_{2}\right\} \\
& \cdot\left\|A y^{n}-A x_{1}^{*}\right\|, \\
& \left\|z^{n}-A x_{1}^{*}\right\| \leq \theta_{2}\left\|A y^{n}-A x_{1}^{*}\right\|,
\end{aligned}
$$

where $\theta_{2}=\left(1 / \sqrt{1+2 \sigma_{2}}\right)\left\{\sqrt{\delta_{2}{ }^{2}-2 \rho_{2} \alpha_{2}+\rho_{2}{ }^{2} \beta_{2}{ }^{2}}+\rho_{2} \xi_{2}+v_{2}\right\}$. Furthermore, in view of Algorithm 5(13c), we have

$$
\begin{aligned}
& \left\|x_{1}^{n+1}-x_{1}^{*}\right\| \leq\left(1-\alpha_{n}\right)\left\|x_{1}^{n}-x_{1}^{*}\right\| \\
& \quad+\alpha_{n}\left[\left\|y^{n}-x_{1}^{*}-\gamma A^{*}\left(A y^{n}-A x_{1}^{*}\right)\right\|\right. \\
& \left.\quad+\gamma\left\|A^{*}\left(z^{n}-A x_{1}^{*}\right)\right\|\right] .
\end{aligned}
$$


Note that $A^{*}$ is a bounded linear operator with $\|A\|=$ $\left\|A^{*}\right\|$ and the given condition on $\gamma$, we get

$$
\begin{aligned}
& \left\|y^{n}-x_{1}^{*}-\gamma A^{*}\left(A y^{n}-A x_{1}^{*}\right)\right\|^{2} \\
& =\left\|y^{n}-x_{1}^{*}\right\|^{2}-2 \gamma\left\langle y^{n}-x_{1}^{*}, A^{*}\left(A y^{n}-A x_{1}^{*}\right)\right\rangle \\
& \quad+\gamma^{2}\left\|A^{*}\left(A y^{n}-A x_{1}^{*}\right)\right\|^{2} \\
& \leq\left\|y^{n}-x_{1}^{*}\right\|^{2}-\gamma\left(2-\gamma\|A\|^{2}\right)\left\|A y^{n}-A x_{1}^{*}\right\| \\
& \leq\left\|y^{n}-x_{1}^{*}\right\|^{2} .
\end{aligned}
$$

And using (29), we have

$$
\begin{aligned}
\left\|A^{*}\left(z_{n}-A x_{1}^{*}\right)\right\| & \leq\|A\|\left\|z_{n}-A x_{1}^{*}\right\| \\
& \leq \theta_{2}\|A\|\left\|A y^{n}-A x_{1}^{*}\right\| \\
& \leq \theta_{2}\|A\|^{2}\left\|y^{n}-x_{1}^{*}\right\| .
\end{aligned}
$$

From (30)-(32),

$$
\left\|x_{1}^{n+1}-x_{1}^{*}\right\| \leq\left[1-(1-\theta) \alpha_{n}\right]\left\|x_{1}^{n}-x_{1}^{*}\right\|
$$

where $\theta=\theta_{1}\left(1+\gamma\|A\|^{2} \theta_{2}\right)$. It follows from the conditions on $\rho_{1}, \rho_{2}$, and $\gamma$ that $\theta \in(0,1)$. Thus, $\left\{(1-\theta) \alpha_{n}\right\} \subset(0,1)$ and $\sum_{n=1}^{\infty}(1-\theta) \alpha_{n}=\infty$ for $\sum_{n=1}^{\infty} \alpha_{n}=\infty$. So it follows from Lemma 13 that $\left\{x_{1}^{n}\right\}$ converges strongly to $x_{1}^{*}$ as $n \rightarrow \infty$. Since $A$ is continuous, it follows from (24), (27), (28), and (29) that

$$
\begin{aligned}
g_{1}\left(y^{n}\right) & \longrightarrow g_{1}\left(x_{1}^{*}\right), \\
y^{n} & \longrightarrow x_{1}^{*}, \\
A y^{n} & \longrightarrow A x_{1}^{*}, \\
g_{2}\left(z^{n}\right) & \longrightarrow g_{2}\left(A x_{1}^{*}\right), \\
z^{n} & \longrightarrow A x_{1}^{*}
\end{aligned}
$$

This completes the proof.

If $h_{i}=0$, then Theorem 14 reduced to the following result of the convergence of Algorithm 6 for SpGQVIP (1a)-(1b).

Corollary 15. For each $i \in\{1,2\}$, let $C_{i}: H_{i} \rightarrow 2^{H_{i}}$ be a nonempty, closed, and convex set-valued mapping, and let $g_{i}: H_{i} \rightarrow H_{i}$ be $\delta_{i}$-Lipschitz continuous such that $\left(g_{i}-I_{i}\right)$ is $\sigma_{i^{-}}$ strongly monotone, where $I_{i}$ is the identity mapping on $H_{i}$. Let $f_{i}: H_{i} \rightarrow H_{i}$ be $\alpha_{i}$-strongly monotone with respect to $g_{i}$ and $\beta_{i}$-Lipschitz continuous. Let $A: H_{1} \rightarrow H_{2}$ be a bounded linear operator and $A^{*}$ be its adjoint mapping. Suppose $x_{1}^{*} \in H_{1}$ is a solution to SpGQVIP (1a)-(1b) and Assumption 9 holds. Then, the sequence $\left\{x_{1}^{n}\right\}$ generated by Algorithm 6 convergences

strongly to $x_{1}^{*}$ provided that the constants $\rho_{i}$ and $\gamma$ satisfy the following conditions:

$$
\begin{aligned}
& \left|\rho_{1}-\frac{\alpha_{1}}{\beta_{1}^{2}}\right| \leq \frac{\sqrt{\alpha_{1}^{2}-\beta_{1}^{2}\left(\delta_{1}^{2}-k_{1}^{2}\right)}}{\beta_{1}^{2}}, \\
& \alpha_{1}>\beta_{1} \sqrt{\delta_{1}^{2}-k_{1}^{2}}, \\
& k_{1}=\left[\frac{\sqrt{1+2 \sigma_{1}}}{\sqrt{1+2 \theta_{2}}}-v_{1}\right], \quad \delta_{1}>\left|k_{1}\right|, \\
& 0<\theta_{2}=\frac{1}{\sqrt{1+2 \sigma_{2}}}\left\{v_{2}+\sqrt{\delta_{2}^{2}-2 \rho_{2} \alpha_{2}+\rho_{2}^{2} \beta_{2}^{2}}\right\}, \\
& \rho_{2}>0, \gamma \in\left(0, \frac{2}{\|A\|^{2}}\right) .
\end{aligned}
$$

If $C_{i}\left(x_{i}\right)=C_{i}\left(\forall x_{i} \in H_{i}\right)$, where $C_{i}$ is a nonempty closed and convex subset of $H_{i}, g_{i}=I_{i}$, and $h_{i}=0$, then Theorem 14 reduces to the following convergence result of Algorithm 7 for SpVIP (2a)-(2b).

Corollary 16. For each $i \in\{1,2\}$, let $C_{i}$ be a nonempty, closed, and convex subset of $H_{i}$. Let $f_{i}: H_{i} \rightarrow H_{i}$ be $\alpha_{i}$-strongly monotone and $\beta_{i}$-Lipschitz continuous. Let $A: H_{1} \rightarrow H_{2}$ be a bounded linear operator and $A^{*}$ be its adjoint operators. Suppose $x_{1}^{*} \in H_{1}$ is a solution to $\operatorname{SpVIP}(2 a)-(2 b)$. Then, the sequence $\left\{x_{1}^{n}\right\}$ generated by Algorithm 7 converges strongly to $x_{1}^{*}$ provided that the constants $\rho_{i}$ and $\gamma$ satisfy the following conditions:

$$
\begin{aligned}
\left|\rho_{1}-\frac{\alpha_{1}}{\beta_{1}{ }^{2}}\right| \leq \frac{\sqrt{\alpha_{1}^{2}-\beta_{1}^{2}\left(1-k_{1}^{2}\right)}}{\beta_{1}^{2}}, & \alpha_{1}>\beta_{1} \sqrt{1-k_{1}^{2}}, \\
k_{1}=\frac{1}{1+2 \theta_{2}}, & k_{1}<1, \theta_{2}=\sqrt{1-2 \rho_{2} \alpha_{2}+\rho_{2}{ }^{2} \beta_{2}{ }^{2}} .
\end{aligned}
$$

\section{Competing Interests}

The authors declare that they have no competing interests.

\section{Acknowledgments}

This work is supported by the National Natural Science Foundation of China under Project no. 11371070.

\section{References}

[1] A. Bensoussan, M. Goursat, and J.-L. Lions, "Controle impulsionn-el et inequations quasi-variationnelles stationnaires," Comptes Rendus de l'Académie des Sciences, Series A-B, vol. 276, pp. A1279-A1284, 1973.

[2] K. R. Kazmi, "Split general quasi-variational inequality problem," Georgian Mathematical Journal, vol. 22, no. 3, pp. 385-392, 2015. 
[3] Y. Censor, A. Gibali, and S. Reich, "Algorithms for the split variational inequality problem," Numerical Algorithms, vol. 59, no. 2, pp. 301-323, 2012.

[4] Y. Censor, T. Bortfeld, B. Martin, and A. Trofimov, "A unified approach for inversion problems in intensity-modulated radiation therapy," Physics in Medicine and Biology, vol. 51, no. 10, pp. 2353-2365, 2006.

[5] Y. Censor and T. Elfving, "A multiprojection algorithm using Bregman projections in a product space," Numerical Algorithms, vol. 8, no. 2-4, pp. 221-239, 1994.

[6] P. L. Combettes, "The convex feasibility problem in image recovery," Advances in Imaging and Electron Physics, vol. 95, no. C, pp. 155-270, 1996.

[7] K. R. Kazmi, "Split nonconvex variational inequality problem," Mathematical Sciences, vol. 7, article 36, 2013.

[8] H.-K. Xu, "Iterative algorithms for nonlinear operators," Journal of the London Mathematical Society. Second Series, vol. 66, no. 1, pp. 240-256, 2002. 


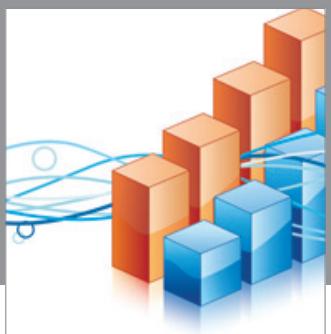

Advances in

Operations Research

vatem alat4

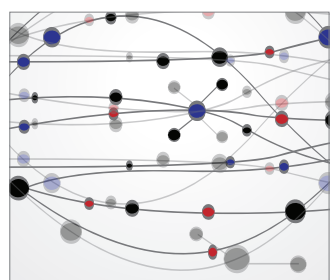

\section{The Scientific} World Journal
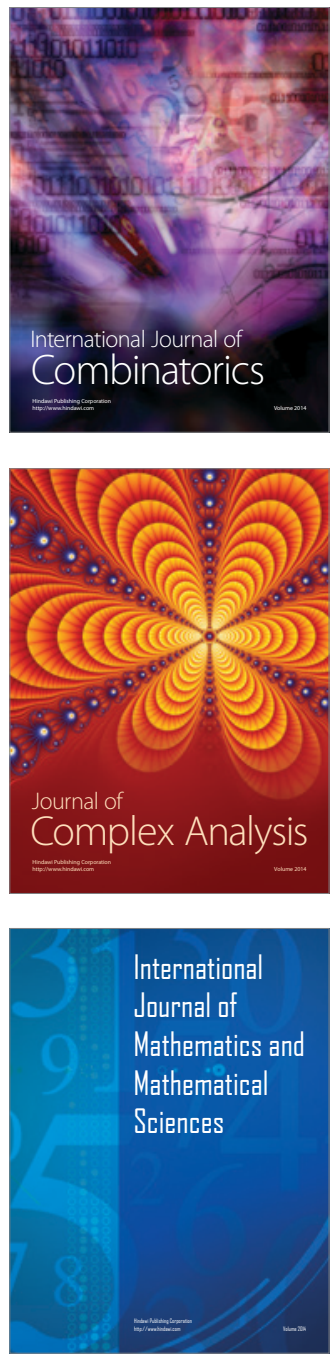
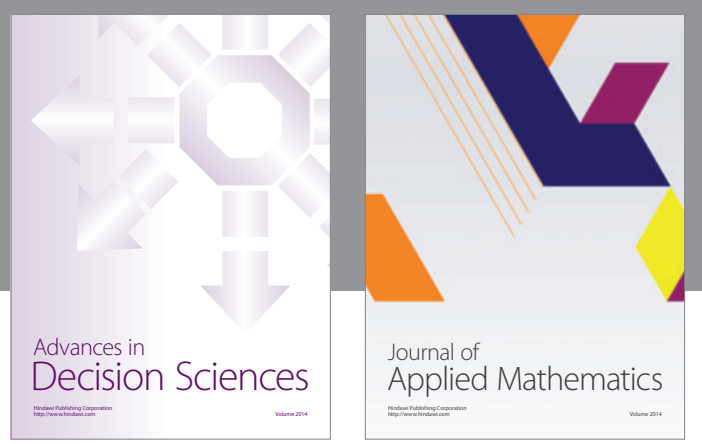

Algebra

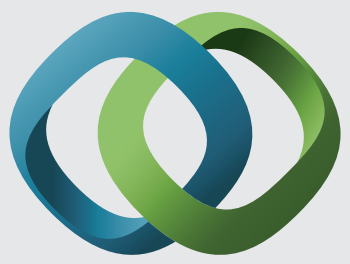

\section{Hindawi}

Submit your manuscripts at

http://www.hindawi.com
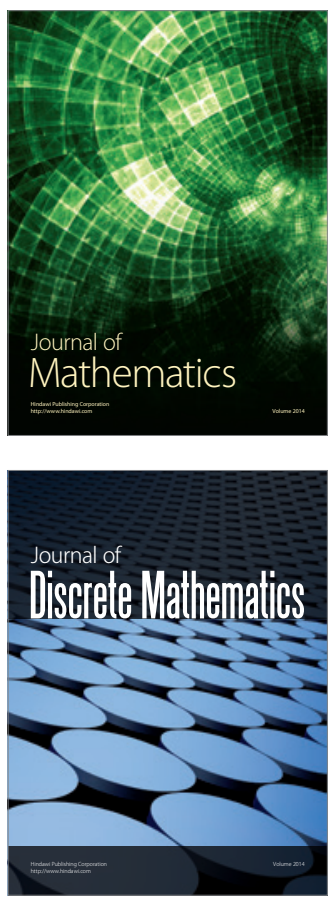

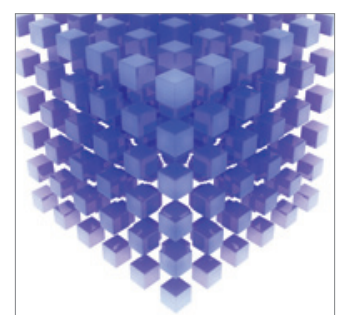

Mathematical Problems in Engineering
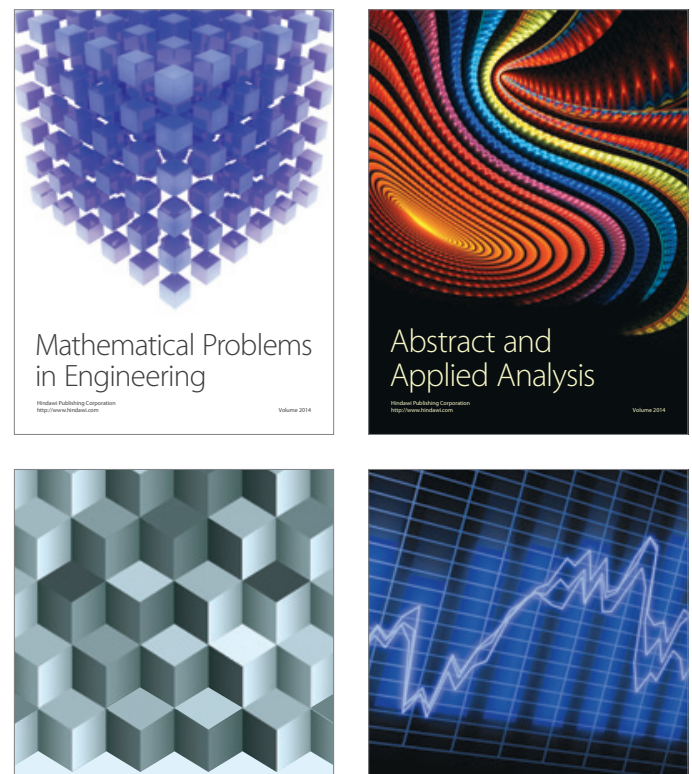

Journal of

Function Spaces

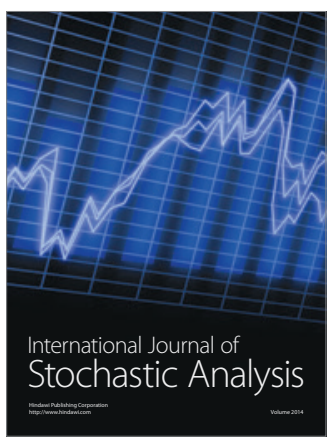

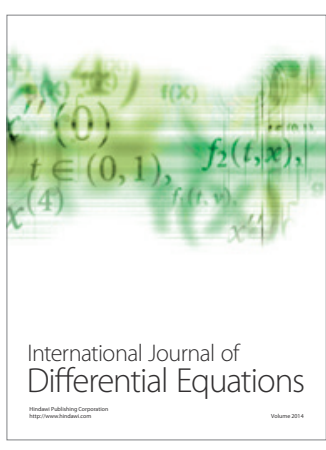
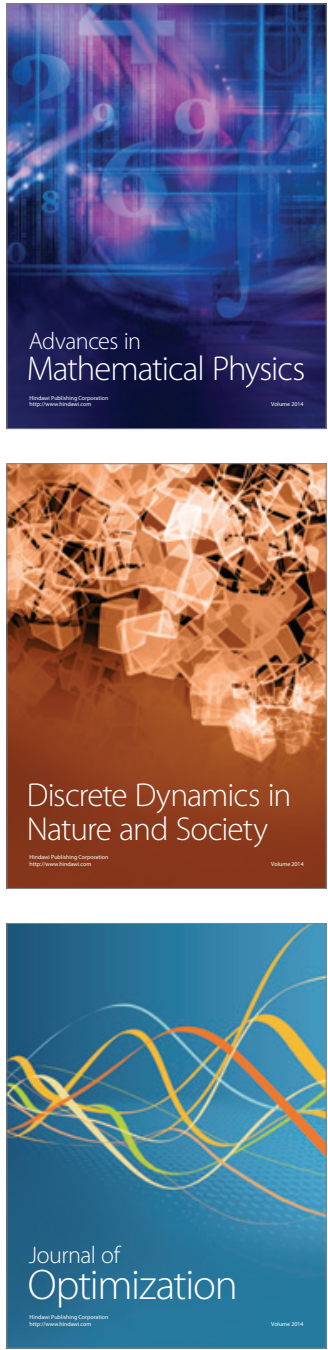